\title{
PROPUESTA BASADA EN MODELOS PARA LA MONITORIZACIÓN REMOTA DE PROCESOS EN LA INDUSTRIA DE PRODUCCIÓN DE PETRÓLEO
}

\author{
Edurne Irisarri ${ }^{1}$, Marcelo V. García ${ }^{1}$, Federico Pérez ${ }^{1}$, Darío Orive ${ }^{1}$, Elisabet Estévez ${ }^{2}$, Marga Marcos ${ }^{1}$ \\ ${ }^{1}$ Universidad del País Vasco, UPV/EHU \\ \{edurne.irisarri, mgarcia294, federico.perez, marga.marcos\}@ehu.eus \\ 2Universidad de Jaén, eestevez@ujaen.es
}

\begin{abstract}
Resumen
Una de las bases sobre las que se asienta la cuarta revolución industrial es la accesibilidad a cualquier información relevante en tiempo real. Esto se alcanza conectando todas las instancias que conforman la cadena de valor. Lo que se persigue, en general, es la gestión de todo el proceso de la cadena de valor, que derivará en una producción más eficiente dando lugar a mejores productos $y$ servicios. Las redes verticales tienen su fundamento en la utilización de sistemas de producción cíberfísicos (CPPSs) y en las organizaciones que posibilitan la integración vertical que va desde los sensores hasta el nivel de empresa de la compañía. Sin embargo, la interoperabilidad global solo se garantiza si se utilizan estándares industriales para modelar las vistas de las partes implicadas y comunicando los dispositivos presentes, diversos y heterogéneos. Este artículo está enfocado al área de la industria de procesos, en particular, a la de producción de petróleo. Para ello se presenta una propuesta de modelado basada en estándares industriales. El objetivo es generar la configuración del servidor OPC UA a partir de los modelos de la planta y de los dispositivos que suministran los datos. Se ha realizado la validación de estos modelos en parte del proceso que desarrolla la compañia Petroamazonas EP de Ecuador.
\end{abstract}

Palabras Clave: Industria de Producción de Petróleo, Industry 4.0, Paradigma conducido por modelos, ISA95, ISA88.

\section{INTRODUCCIÓN}

La industria de producción de petróleo, tanto en el caso on-shore como en off-shore, se caracteriza por estar formada por una estructura distribuida en múltiples ubicaciones geográficas muy distantes entre sí. En este tipo de industria de procesos se da la particularidad de que muchas de las operaciones se llevan a cabo hoy en día todavía de un modo local y, en no pocos casos, incluso manualmente. Esta realidad supone, entre otras desventajas, unos elevados costes para las compañías así como una lenta respuesta ante variaciones de demanda.

Por tanto, existe una clara necesidad de mejorar la gestión del proceso distribuido, adaptando el sistema de control a las características propias de múltiples ubicaciones para las partes del proceso, y ofreciendo la posibilidad de monitorizar remotamente y dar respuesta a las demandas en tiempo real.

Para alcanzar esto, un primer paso es contar con la posibilidad de monitorizar remotamente el estado y evolución del proceso. En este sentido, la industria de producción de petróleo podría beneficiarse de determinadas propuestas impulsadas desde Industry 4.0 [3]. Esta iniciativa promueve, por un lado, la introducción de conceptos en fábrica como son IoT (Internet of Things) y sistema cíber-físico, que hacen posible la transmisión de los datos que se capturan en el proceso y los hace accesibles a través de la nube. Otro modo de transmitir los datos pre-procesados podría ser adquiriendo la información de los cientos de transmisores ya instalados en la planta a través de sensores inteligentes (smart sensors). Por otra parte, Industry 4.0 insiste en la importancia de utilizar estándares consolidados para representar formalmente la información que se captura, guarda y transmite, así como para especificar las tecnologías de comunicación para su transmisión.

Considerando las necesidades identificadas en el sector de producción del petróleo, este artículo presenta el trabajo que se está realizando en relación con el reto que supone la monitorización remota de este proceso particular. Se presenta aquí una propuesta genérica que facilita el acceso remoto a los datos del proceso, alcanzando una integración vertical desde las áreas de empresa o negocio hasta el nivel de producción en planta. Para este propósito y siguiendo la tendencia recomendada por Industry 4.0, se han seleccionado los estándares, OPC UA (IEC 62541) [9] e ISA 95 (IEC 62264) [2] e ISA 88 
(IEC 61512) [1] como columna vertebral para la comunicación y modelado de planta respectivamente.

En esta línea, pueden destacarse proyectos de investigación que utilizan el estándar ISA95 para la definición de arquitecturas. Es el caso de HoVer [4], que aborda la integración de los flujos de información horizontal y vertical, donde los escasos aspectos de la integración vertical que se abordan lo hacen utilizando conceptos de ISA95, aunque únicamente como extensión del núcleo, centrado en la horizontal. Otro ejemplo es [5] que aborda la monitorización desde un entorno orientado a servicios (SOA) y con organización basada en Redes de Petri de alto nivel. De ISA95, en este caso, solo se utiliza el modelo que identifica los niveles de la empresa. En cuanto a los otros estándares, mencionados, en [6] se propone una arquitectura abierta basada en ISA88 para fabricación flexible. Para ello, proponen una aproximación modular que minimiza los esfuerzos de programación en el sistema de automatización que exige un nuevo producto. Además, las interfaces entre los módulos se basan en OPC UA. Sin embargo, la integración vertical no está entre sus objetivos.

En trabajos anteriores [7] los autores presentaron una arquitectura general para la monitorización remota. Ésta consiste en una propuesta basada en modelos donde se identifican los diferentes modelos necesarios para alcanzar la integración vertical. Este artículo va más allá, pues aplica dicha arquitectura genérica al caso particular de la industria de producción del petróleo. Para ello se definen unos meta-modelos específicos -basados en estándares industriales (ISA95 e ISA88)- para el proceso de producción y para los dispositivos inteligentes encargados de suministrar la información.

El orden que sigue este artículo es el siguiente: en el apartado 2 se presenta el escenario general, en el que se incluye una breve descripción del proceso de producción del petróleo. El apartado 3 describe el meta-modelo para el proceso de producción de petróleo cuya implementación para el caso particular de la compañía Petroamazonas EP [8] queda ilustrada en el apartado 4. Por último, en el apartado 5 se extraen ciertas conclusiones y se introducen trabajos futuros o ya en marcha.

\section{ESCENARIO GENERAL}

La base de este trabajo es la arquitectura presentada en [7]. La Figura 1 representa el escenario general para la monitorización del proceso. En él se destacan los meta-modelos que se proponen en este artículo. En particular:
- El mundo físico, entendido como proceso de producción, se define por el Modelo del Proceso de Producción (Production Process, PPM). Se especifica -siguiendo los estándares ISA95 e ISA88- desde dos puntos de vista:

oLa planta física, incluyendo las variables del proceso accesibles, se define por medio del también llamado Modelo de Topología de la Planta (Plant Topology Model, PTM).

○Los dispositivos proveedores de los datos, como son los controladores y/o los dispositivos inteligentes que ofrecen datos del proceso, y sus características se definen en el llamado Modelo de Dispositivos Inteligentes de Planta (Plant Intelligent Devices Model, PIDM).

La arquitectura define también otros dos modelos:

- El Modelo de Información de Planta (Plant Information Model, PIM), se corresponde con los requisitos de monitorización del usuario que pueden definirse desde el PTM especificando las variables del proceso así como el tipo de acceso.

- El Modelo de Intercambio de Información (Information Exchange Model, IEM), procesa los requisitos del usuario para la monitorización y es el encargado del acceso a los dispositivos de datos del proceso adecuados.

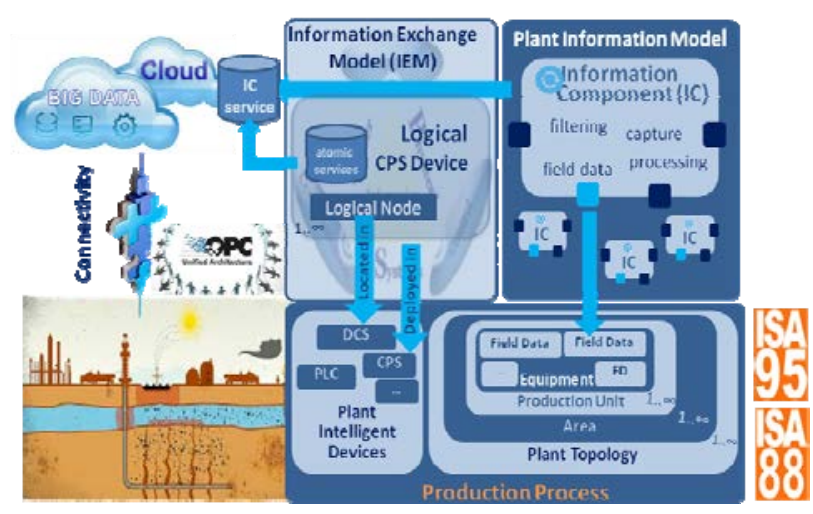

Figura 1: Escenario general para el proceso de monitorización

Este artículo se centra concretamente en la definición del PPM completo para el caso del proceso de producción de petróleo, tomando como base los estándares previamente citados.

Una característica común a tener en cuenta en el sector de la producción del petróleo es que las compañías se encuentran geográficamente distribuidas, con diferentes ubicaciones para las distintas partes del proceso. Por ello, es de vital importancia disponer de un acceso remoto al proceso con el fin de que se pueda monitorizar completamente o tomar decisiones que afecten a las 
diferentes ubicaciones. Así, las compañías de producción de petróleo suelen estar compuestas por múltiples bloques en los que se realiza la extracción del crudo y se obtienen el petróleo, gas y agua tras la separación trifásica posterior. Cada bloque, por tanto, suele estar formado por un módulo de extracción del crudo y otro de separación a partir de la cual se obtienen el petróleo, el gas y el agua. Concretamente, el crudo se extrae del fondo de los pozos (wells) mediante bombeo y una vez en la superficie, se envía al conjunto de tuberías de conducción del crudo (manifold). Es precisamente aquí, en la conducción, donde se recoge el crudo procedente de todos los pozos agrupados en el well pad (Ver unidades de Extraction y Transportation de la Figura 2).

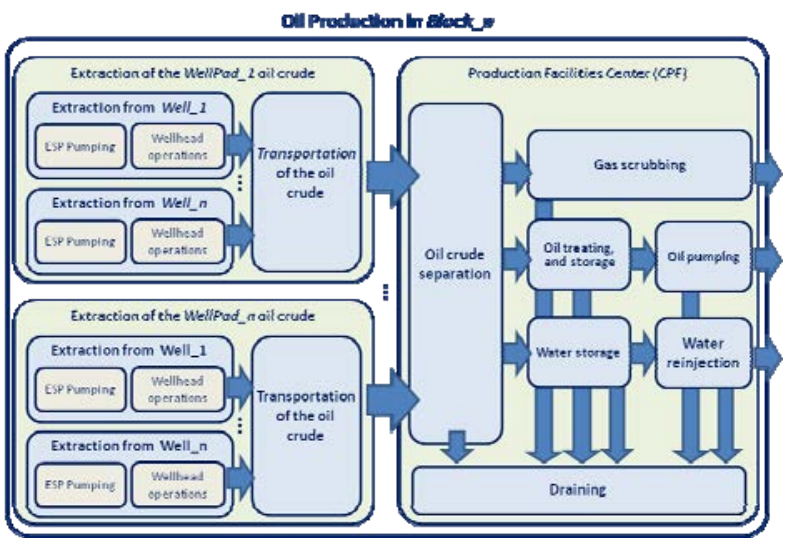

Figura 2: Descripción del proceso de producción de petróleo

El proceso de extracción del crudo finaliza cuando este es enviado al Centro de Facilidades de Producción (Production Facilities Center, CPF). El CPF está formado por un depósito al que llega el crudo procedente de todos los well pads del bloque. Tal como se recoge en la Figura 2, es a partir de este depósito donde comienzan a obtenerse el petróleo en sí, el gas y el agua. El petróleo que se genera aquí se trata, almacena y bombea (oil treatment and storage, oil pumping) para posteriormente enviarse a otras compañías donde se continuará con su refinado. Simultáneamente, parte del gas separado se quema y el resto se expulsa mediante gasoductos (gas scrubbing). El agua obtenida se almacena, trata y reinyecta en los pozos (water storage, water reinjection). Por último, se van drenando las impurezas y otros materiales de desecho que arrastran los fluidos (draining) a lo largo de todas las fases del CPF.

\section{META-MODELO DEL PROCESO DE PRODUCCIÓN DEL PETRÓLEO (PPM)}

El Meta-Modelo del Proceso de Producción del Petróleo, PPM, está formado por dos partes principales. Por un lado, el Meta-Modelo PTM, que define la parte de los componentes físicos de la planta de producción de petróleo, esto es, el conjunto de procesos de producción que agrupan los datos accesibles de cada proceso. El PTM debería ser lo suficientemente genérico como para describir cualquier proceso de producción de petróleo pero que permita ser particularizado a las especificidades de una empresa concreta. Independientemente del número de niveles de la jerarquía, el nivel inferior se refiere al conjunto de procesos caracterizados por un conjunto de variables físicas del proceso. Estas variables se caracterizan por sus propiedades (como son, entre otras, la magnitud física, las unidades de ingeniería o el rango de los posibles valores que puede tomar). Por otra parte, el Meta-Modelo PIDM describe la parte correspondiente a los dispositivos con capacidad de procesamiento -controladores de proceso o dispositivos inteligentes- que pueden actuar en la arquitectura como proveedores de datos. Los siguientes sub-apartados describen los respectivos meta-modelos conformes a los estándares ISA95 e ISA88 que se ajustan a las especificidades de la industria de producción de petróleo.

\subsection{META-MODELO DE TOPOLOGÍA DE PLANTA (PTM)}

El estándar ISA95 y el modelo físico de ISA88 proponen un modelo jerárquico donde se caracteriza el rol que toma el equipamiento en los procesos de producción. Partiendo de estos modelos generales, válidos tanto para procesos por lotes, continuos como discretos, se presentan a continuación unos modelos adaptados al caso de proceso continuo, propio del sector de producción del petróleo:

- Empresa (Enterprise). La empresa es la responsable de determinar qué productos se van a fabricar, en qué sedes o ubicaciones y, en general, cómo van a ser fabricados. En el caso del campo de producción de petróleo, se considera como tal a la Compañía Petrolera.

- Sitio (Site). Una ubicación geográfica con competencias considerables de producción se identifica tradicionalmente como site. De hecho, la empresa en sí está constituida por un grupo de sitios o sedes. Cada sitio se planifica y organiza, a su vez, en células, líneas o unidades distribuidas en áreas. Los sitios suelen tener atribuidas unas competencias de fabricación claras. En el caso particular de las empresas de producción de petróleo, el equivalente al concepto estándar de Sitio sería el Bloque (Block). 


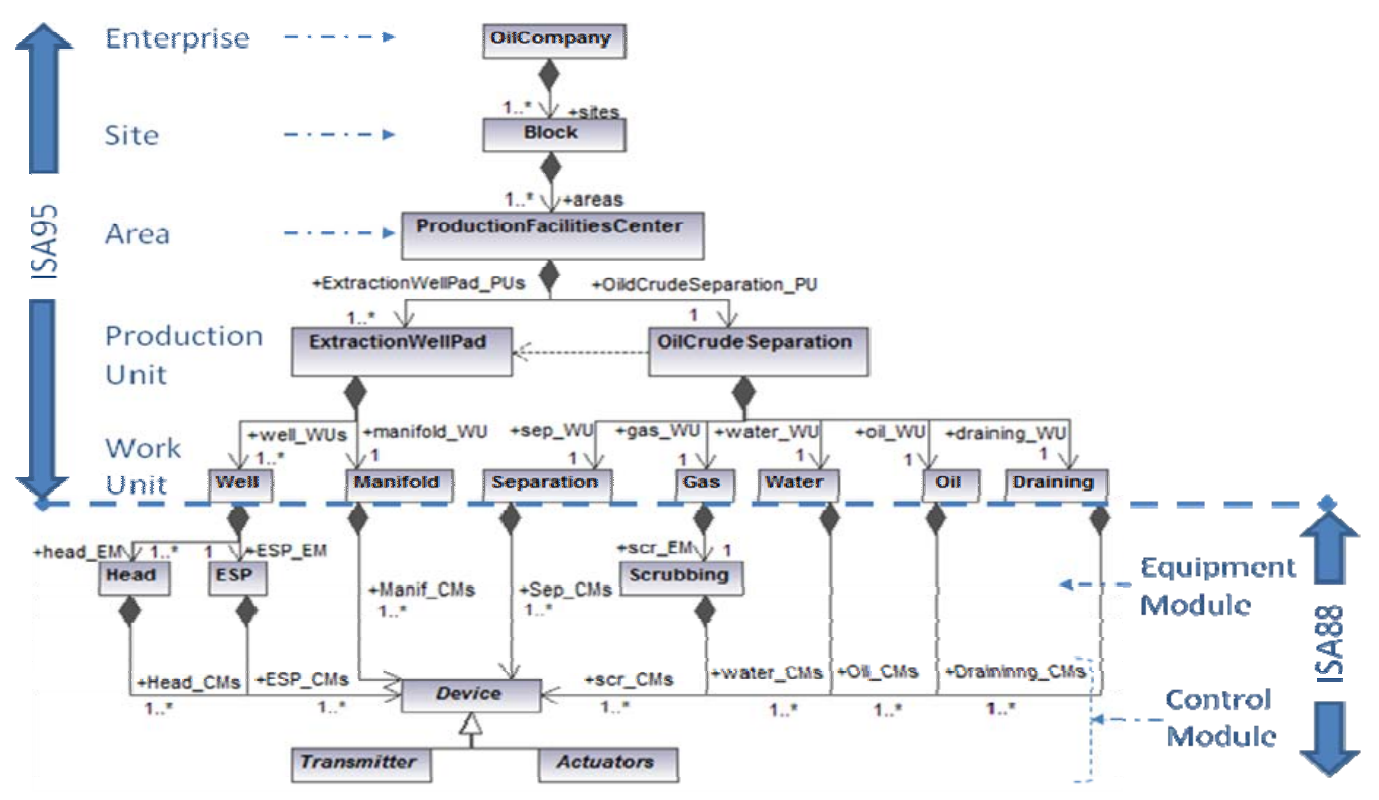

Figura. 3: Léxico y sintaxis del Meta-Modelo de Topología de Planta

- Área (Area). Las principales competencias de producción y ubicaciones geográficas dentro de un sitio se definen como áreas. Las áreas generalmente cuentan también con competencias de fabricación claramente definidas. Están, a su vez, formadas por elementos que llevan a cabo las diferentes funciones de fabricación. Dependiendo del tipo de proceso, estas áreas incluyen una combinación de líneas de producción (si actividad basada en operaciones discretas), de unidades de producción ( $\mathrm{si}$ operaciones continuas) o células de proceso (si procesos por lotes). El concepto de área en el proceso de producción de petróleo podría presentarse por el Centro de Facilidades de Producción $(C P F)$. Por tanto, cada Bloque de una Compañía de Producción de Petróleo estará formado por un conjunto de CPFs, equivalente al concepto estándar de área.

- Unidad de Producción (Production Unit, PU). Una unidad de producción generalmente engloba todo el equipamiento que se necesita en un segmento de producción continua que opera de manera relativamente autónoma. Ejemplos de ello son, entre otros, los módulos de equipamiento, sensores o actuadores, aunque estos últimos quedan fuera del alcance de ISA95. Por tanto, en el caso de la Producción del Petróleo, como unidades de producción pueden identificarse dos: la PU de Extracción del Well Pad y la PU de Separación del Crudo (ver Figura 3).

- Unidad de Trabajo (Work Unit, WU). Identifica la capacidad de procesamiento principal de la familia de productos. Las WUs son el nivel inferior del equipamiento que participa en las operaciones propias de los niveles 3 y 4 de los procesos de fabricación. Así, cada PU de extracción del wellpad se caracteriza por dos tipos de WU: (1) Los pozos de los que se extrae el crudo y (2) el conjunto de conductos (manifold) que transportan dicho fluido a la PU de separación del crudo. Del mismo modo, la PU de separación del crudo está formada por cinco WUs: (1) Separación, (2) Gas, (3) Agua, (4) Petróleo y (5) Drenaje (Ver Figura 2).

- Módulo de Equipamiento (Equipment Module, $E M)$. Físicamente, el EM puede estar formado por módulos de control y otros módulos de equipamiento subordinados. Un EM, a su vez, puede ser parte de una unidad o de un grupo de equipos autónomos dentro de una WU. Un EM es la combinación de todos los equipos físicos de control y procesamiento necesarios para llevar a cabo esas actividades. Suelen estar centrados en un equipo de procesamiento. En el caso del proceso de producción del petróleo se ha identificado un conjunto de EMs. Tal es el caso de la WU pozo, que está formada por dos EMs: (1) la cabecera del pozo (Head) y (2) el sistema de bombeo electrosumergible $(E S P)$.

- Módulo de Control (Control Module, CM). Un $\mathrm{CM}$ es típicamente una colección de sensores, actuadores y otros dispositivos participantes en el control, así como equipos de procesamiento asociados que, desde el punto de vista del control, operan como una entidad simple. Un CM, a su vez, puede estar formado por otros módulos de control. Algunos ejemplos de CMs en el caso del proceso de producción de petróleo son las Bombas (Pumps), Drives, Válvulas (Valves), diferentes Transmisores e indicadores (Indicating Transmitters) como por ejemplo de presión -PIT-, de temperatura -TIT-, de nivel -LIT-, de caudal -FIP-, de presión diferencial -PDIT-, o transmisores simples (Transmitters) de 
corriente -CT-, de tensión -VT- o TT, PT, FT. La Figura 3 corresponde al Meta-Modelo que se propone para PTM. La Figura 4 muestra las principales características de los Transmitters como son un identificador, el rango de valores, las unidades de ingeniería y el número de serie del correspondiente dispositivo físico (physical asset).

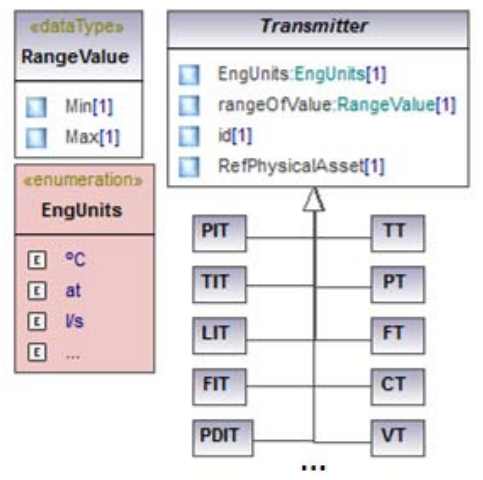

Figura 4: Caracterización de los Transmisores

\subsection{META-MODELO DE DISPOSITIVOS INTELIGENTES DE PLANTA (PIDM)}

Los dispositivos que actúan como proveedores de datos se caracterizan por la información necesaria para acceder a los datos de proceso que proporcionan. Abarcan desde los PLCs, DCSs, sistemas embebidos, controladores industriales, sensores inteligentes hasta cualquier otro dispositivo que tenga acceso a las variables del proceso. Para definir estos dispositivos se ha optado también por utilizar el modelo de equipamiento que propone el estándar ISA95.

Las propiedades de cada dispositivo suministrador son aquellas que especifican la comunicación con los otros componentes de la red (del mismo nivel o de niveles superiores), y los datos del proceso que proporcionan. En la Figura 5 se muestran estas propiedades.

En cuanto a los aspectos de la comunicación, cada suministrador de datos se caracteriza por su dirección (address) y propiedades del protocolo (protocol) de comunicación que utilizan para suministrarlos. Por otra parte, cada dato al que se accede (Data) se caracteriza por un nombre (name), tipo (Type), tamaño (size), dirección (address) y la referencia al dispositivo físico (refDeviceID) del que lo recibe el dispositivo inteligente.
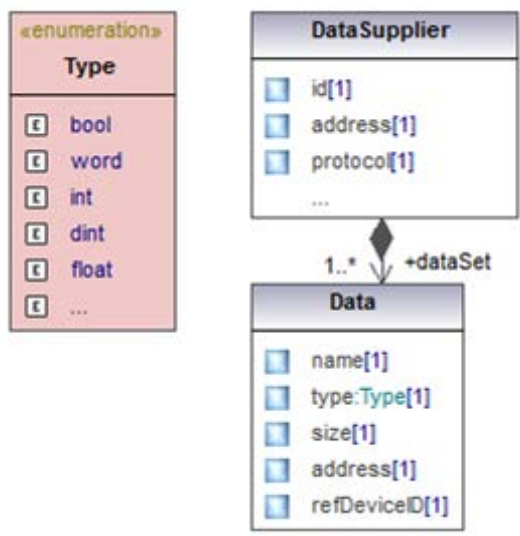

Figura 5: Meta-modelo mínimo para los dispositivos inteligentes capaces de suministrar datos

\section{CASO DE ESTUDIO: PETROAMAZONAS EP}

El caso de estudio se centra en Petroamazonas EP [8], empresa pública ecuatoriana dedicada a la exploración y producción de hidrocarburos. Está a cargo de la operación de 20 Bloques, ubicados en la cuenca Oriente y en la zona del Litoral de Ecuador. Con objeto de simplificar el caso de estudio, se ha modelado únicamente una parte de, en concreto, el Bloque 18. En este Bloque existen en la actualidad 4 wellpads y cada uno de ellos agrupa 30 pozos. El ejemplo tiene por objeto definir una parte del proceso, en concreto la extracción del crudo de uno de los pozos de un wellpad, y más en particular, se refiere al pozo $P A A-001$ cuyo esquema se muestra en la Figura 6.

La cabecera del pozo (wellhead), identificada con $P A A-01-01$, dispone de dos transmisores indicadores: el TIT-WPAA01-01 que indica la temperatura y el PIT-WPAA01-01 la presión del crudo extraído. Asimismo, cuenta con una válvula de emergencia de producción, la $S D V$-WPAA01-01.

Por otra parte, en relación con el sistema de bombeo electro-sumergible BESPA1, que se corresponde con el elemento $M$ de la Figura 6, se han de considerar dos transmisores de presión: el PT-BESPA1-01 para la presión de entrada (intake) y el PT-BESPA1-02 para la presión de descarga (exhaust); tres transmisores de temperatura: el TT-BESPA1-01 para la temperatura del fluido, el TT-BESPA1-02 para la del motor y el TT-BESPA1-03 para la del fluido de salida; un sensor que mide la corriente del motor, CT-BESPA1-01; la tensión del motor se mide mediante $V T-B E S P A 1-01$ y el FT-BESPA1-01 indica el valor de la frecuencia del variador de frecuencia, VSD. 
Asimismo, cabe destacar que el núcleo del sistema de bombeo electro-sumergible lo conforman el motor $M-W P A 01-1$, la bomba $P-W P A A 01$ y el variador de frecuencia $V S D-W P A A 01$.

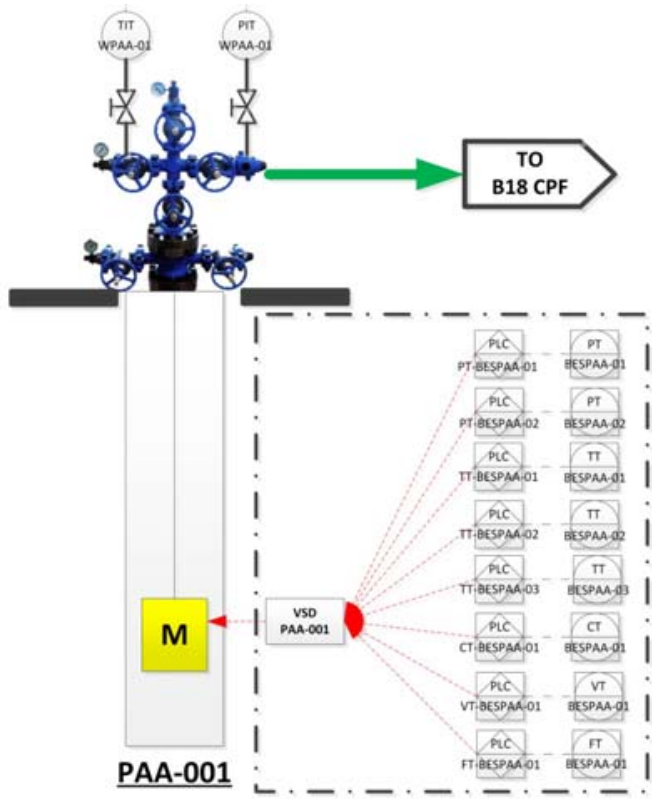

Figura 6: Diagrama P\&ID del sistema de bombeo y extracción del crudo del pozo

Por otra parte, la Figura 7 muestra el esquema del sistema de comunicaciones que se utiliza actualmente entre los módulos de extracción y de separación y los sistemas de monitorización.

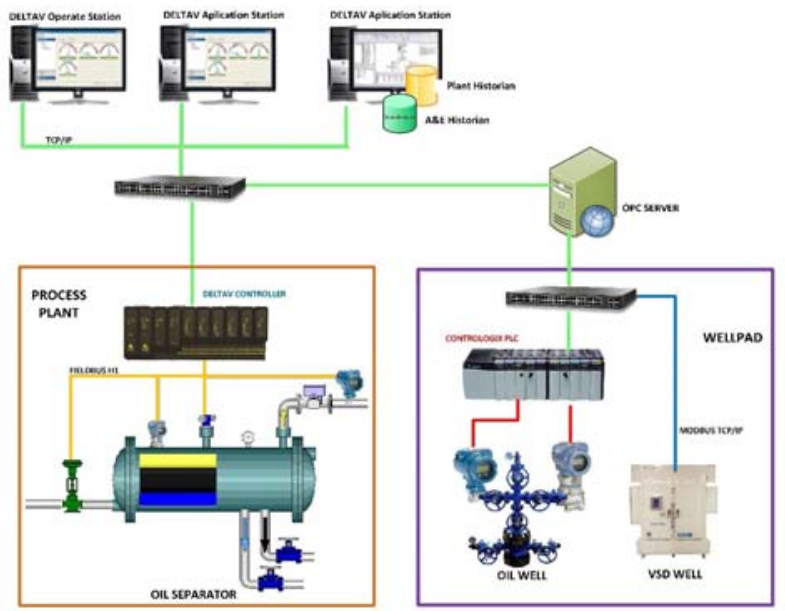

Figura 7: Sistema de comunicación actual entre los módulos de separación y extracción con los sistemas de monitorización (dispositivos inteligentes)

Toda esta información que describe la topología de la planta y los dispositivos inteligentes se guarda en documentos con notación de lenguaje de marcado (Markup Language, ML). Estos documentos siguen su correspondiente schema XML -PTMM.xsd y PIDMM.xsd respectivamente- que son implementaciones basadas en XML de los metamodelos presentados en el apartado anterior. En la Figura 8 se puede observar una parte del modelo PTM aplicado al caso de estudio, y una muestra de la parte correspondiente al modelo PIDM en la Figura 9.

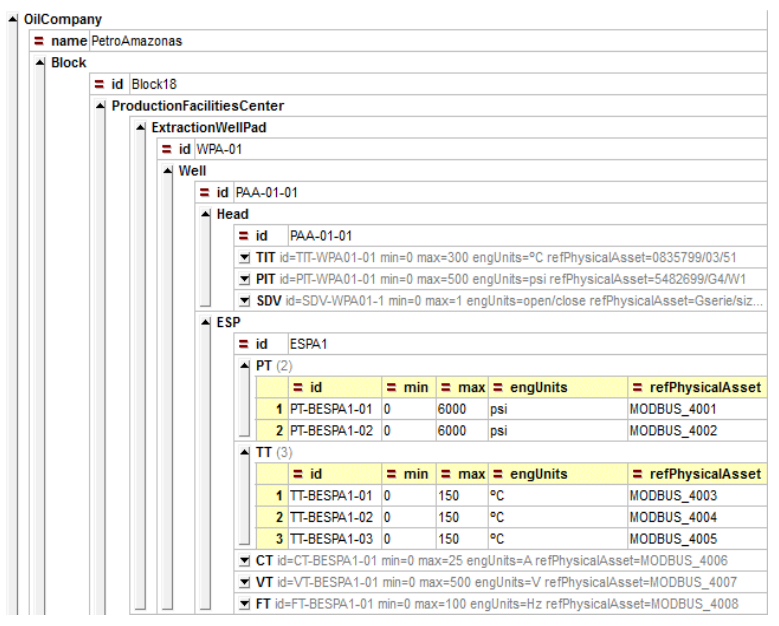

Figura 8: Ejemplo de modelo de Topología de Planta

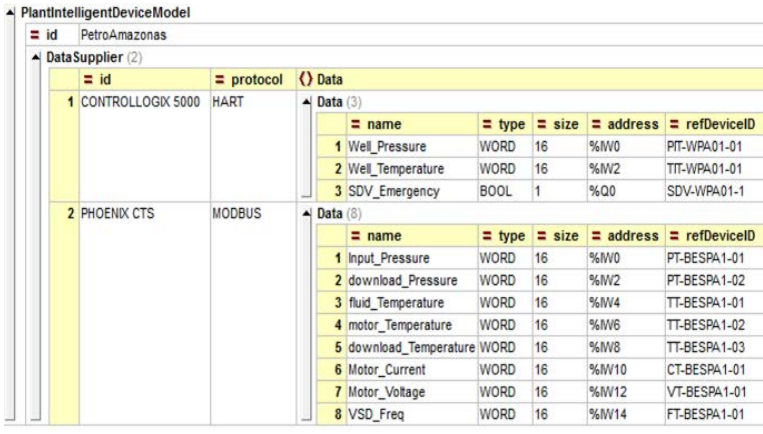

Figura 9: Ejemplo de modelo de Dispositivos Inteligentes de Planta

\section{CONCLUSIONES Y TRABAJOS FUTUROS}

Este trabajo es un primer paso hacia la definición completa de una arquitectura basada en modelos para monitorizar el proceso de producción del petróleo que, utilizando estándares industriales consolidados (ISA95 e ISA88), puede ser la base para una integración "sin costuras" en el sector de la industria de producción de petróleo.

Aquí se ha especificado la información que necesita el servidor OPC UA para dar respuesta a los requisitos de clientes remotos relativas a la monitorización del proceso de producción de petróleo. Como próximos pasos se contemplan, por 
un lado, la definición de los modelos restantes para completar la arquitectura (el PIM y el IEM), así como la generación automática del fichero de configuración del servidor OPC UA y el diseño de la aplicación para la monitorización.

\section{Agradecimientos}

Este trabajo ha sido financiado por el MCYT y FEDER del Gobierno de España bajo el proyecto DPI2015-68602-R y por la Universidad del País Vasco (UPV/EHU) en el marco del proyecto UFI11/28.

\section{Referencias}

[1] ANSI/ISA-88.01-1995, http://www.gmpua.com/GAMP/ISA-88.pdf. Último acceso Junio 2016.

[2] ISA 95, [online] https://isa-95.com/. Último acceso Junio 2016.

[3] Jazdi, N., (2014) "Cyber physical systems in the context of Industry 4.0", Proc. of the IEEE Int. Conf. on Quality and Testing, Robotics, pp. 2-4.

[4] Mazak, A., Huemer, C., (2015) "HoVer: A modeling framework for horizontal and vertical integration", Actas de $13^{\text {th }}$ IEEE Int. Conf. on Industrial Informatics (INDIN), Cambridge.

[5] Nagorny, K., Harrison, R., Colombo, A.W. y Kreutz, G., (2013) "A formal engineering approach for control and monitoring systems in a service-oriented environment", Actas de $11^{\text {th }}$ IEEE Int. Conf. on Industrial Informatics (INDIN), Bochum.

[6] Neugschwandtner, G., Reekmans, M., y Van der Linden, D., (2013) "An open automation architecture for flexible manufacturing," Actas de $18^{\text {th }}$ IEEE Int. Conf. on Emerging Technologies and Factory Automation (ETFA), Cagliari.

[7] Perez, F., Irisarri, E., Orive, D., Marcos, M., y Estevez, E., (2015) "A CPPS Architecture approach for Industry 4.0", Actas de $20^{\text {th }}$ IEEE Int. Conf. on Emerging Technologies and Factory Automation (ETFA), pp. 1-4.

[8] Petroamazonas EP, [página web] http://www.petroamazonas.gob.ec/. Último acceso Junio 2016.
[9] Van Der Linden, D., Mannaert, H., Kastner, W., Vanderputten, V., Peremans, H., y Verelst, J., (2011) "An OPC UA interface for an evolvable ISA88 control module", Actas de 16th Conf. on Emerging Technologies and Factory Automation (ETFA). 